دراسة تأثير تطور التربة ومحتوى أكاسيد الحديد في خصائص الثحنات الأئمة على سطوح الطين لبعض الترب

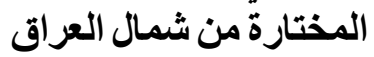

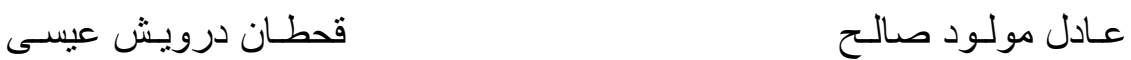

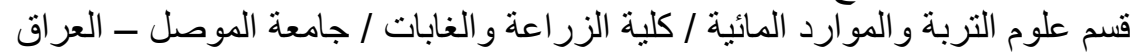 \\ E-mail: Adel_mawlood@yahoo.com
}

\begin{abstract}
الخلاصة
أجريت الدر اسة على تسعة عينات من الطين، جمعت من ثناثة ترب مختلفة من شمال العراق (مخمور

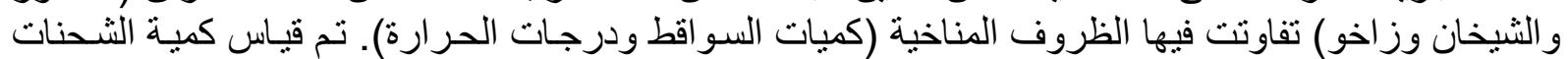

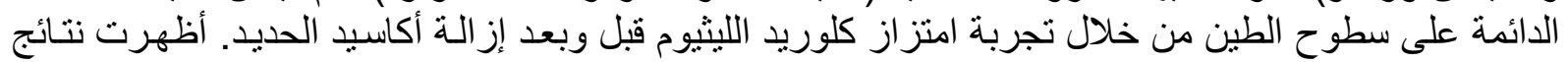

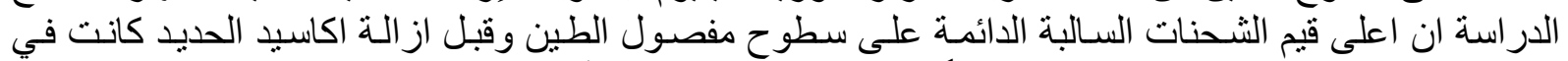

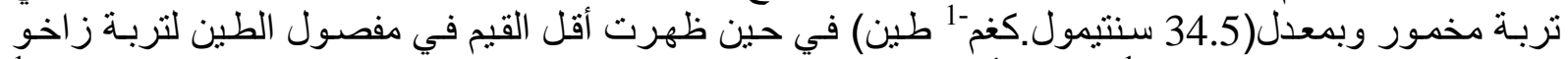

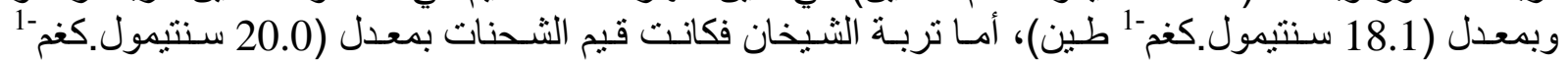

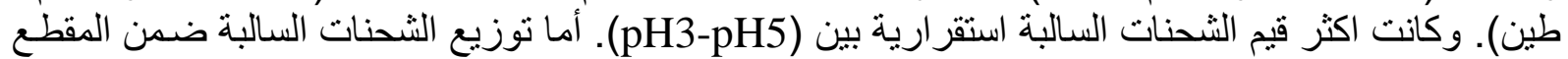

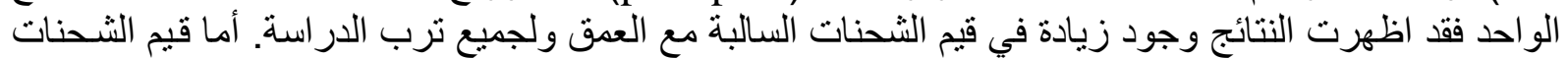

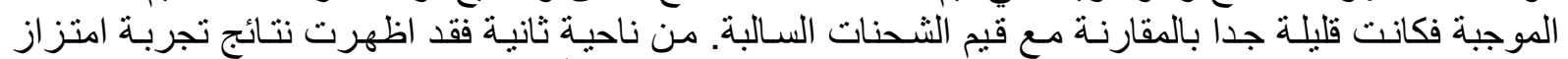

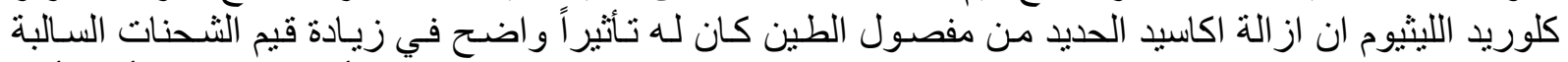

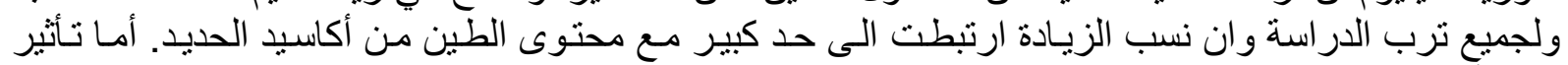

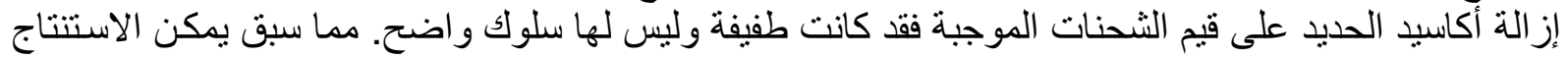

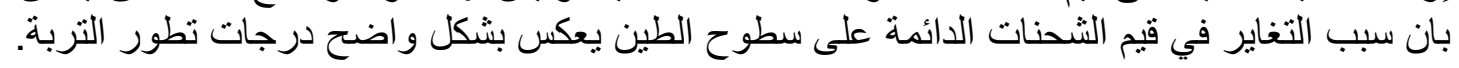
الكلمات الدالة: الثحنات السطحية الموجبة، الثحنات السطحية السالبة، أكاسيد الحديد.

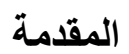

تاريخ تسلم البحث: 2012/12/6 ، وقبوله: 2013/2/18.
\end{abstract}

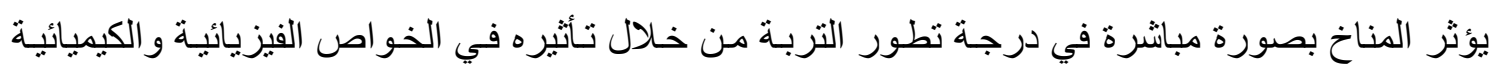

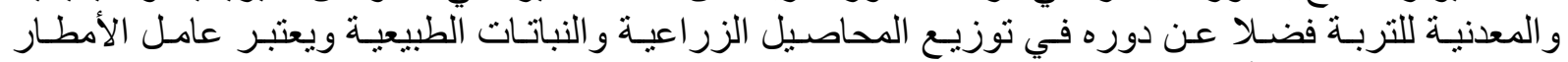

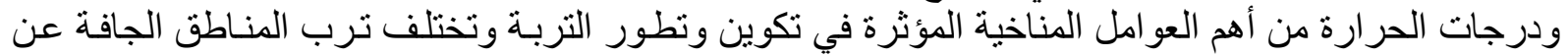

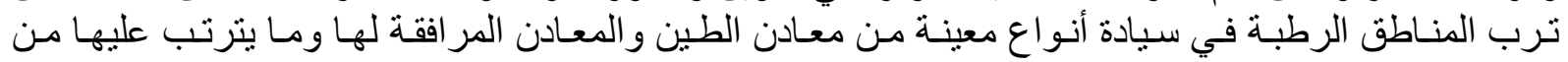

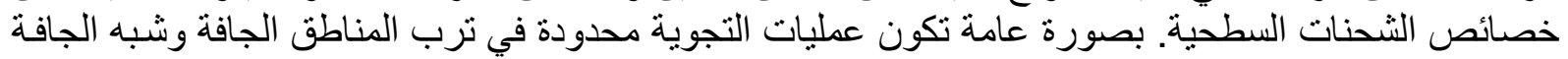

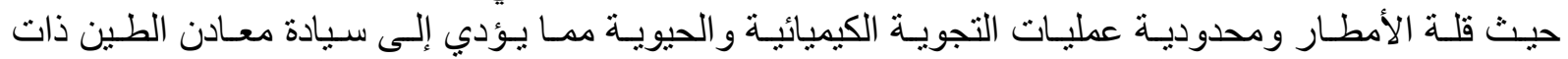

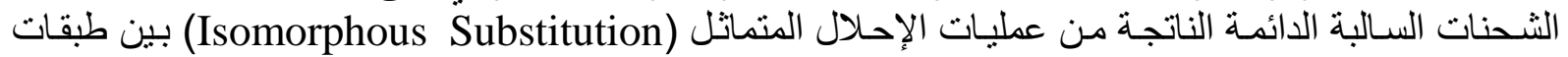
معادن الطين، وبالتالي زيادة التبادل الكاتيوني (Rhoades ،

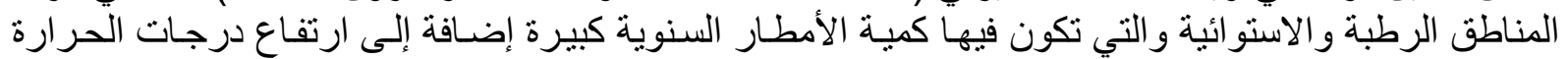

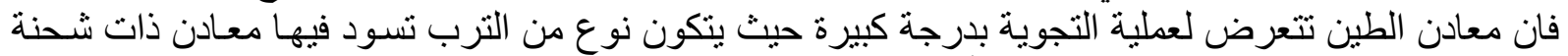

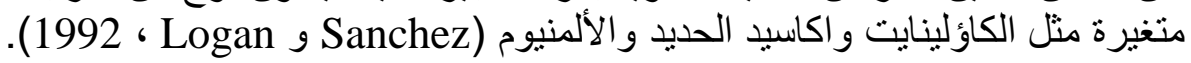

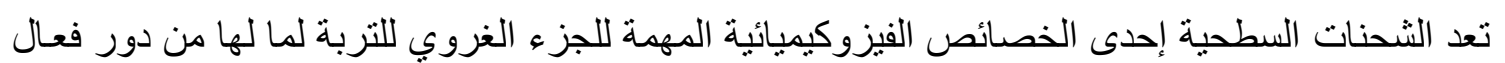

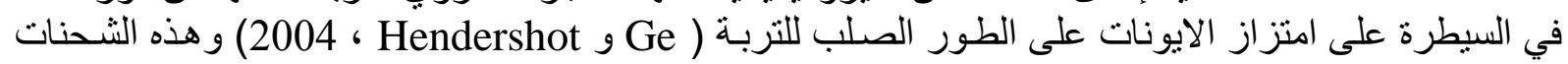

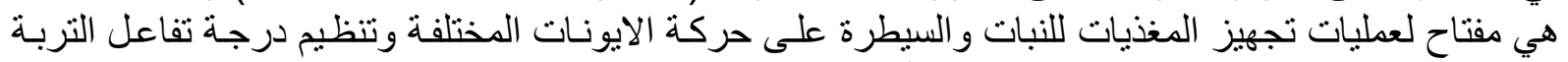

1994, Karlen and Stott)

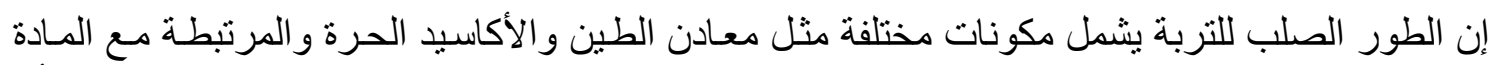

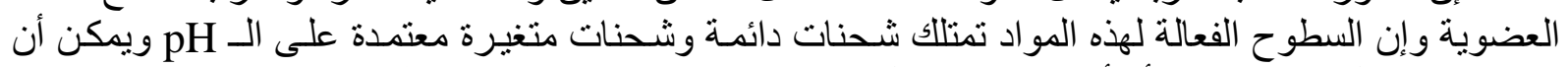

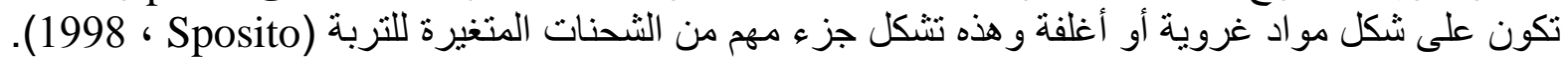


هناللك ثلاثة مصادر اساسية للشحنة على سطوح الطين و الجزء الغروي و هي:

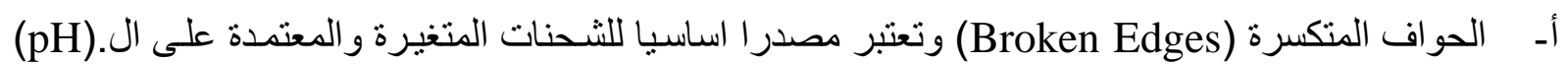

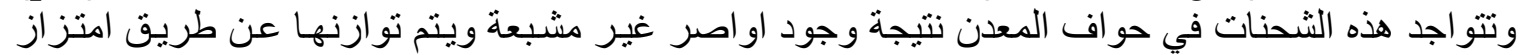

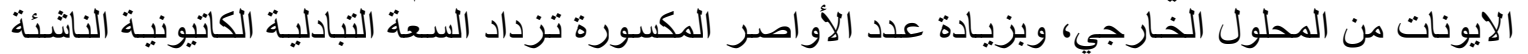

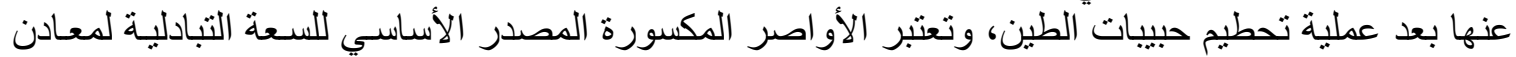

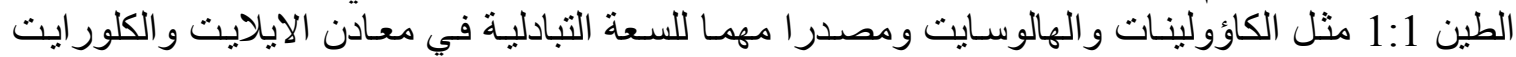

.(Sparks,1995)

بـ الإحلال المتماثل (Isomorphous Substitution) و هو إحلال كاتيونـات ذات تكافؤ أقل محل كاتيونـات

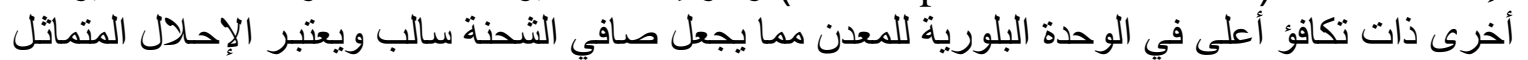

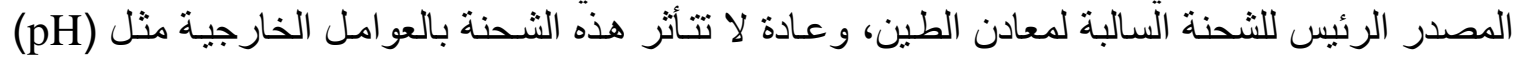

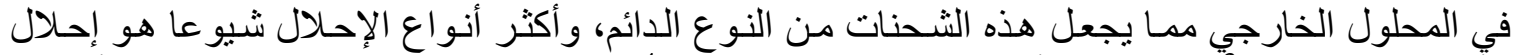
الألمنيـوم الثنلاتي المغنسيوم الثنائي (

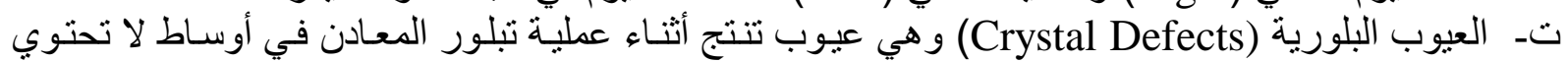

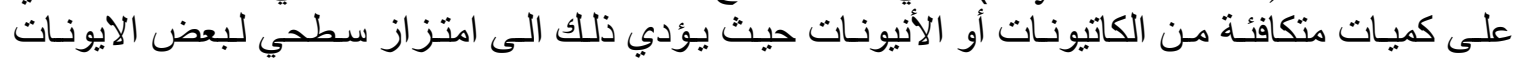

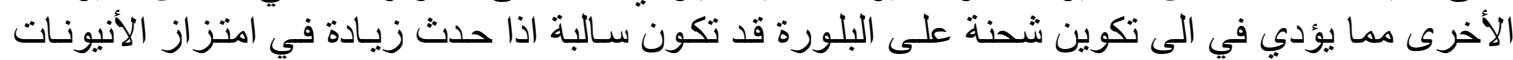

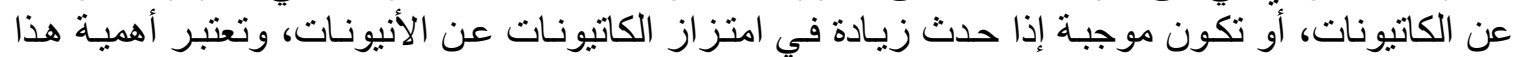

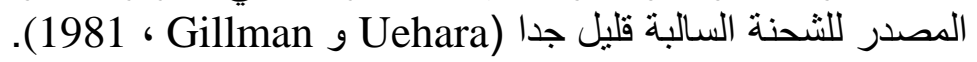

ان الهدف من هذا البحث هو التعرف على مدى تأثثر كمية أكاسيد الحديد الكلية على كمية الثحنات

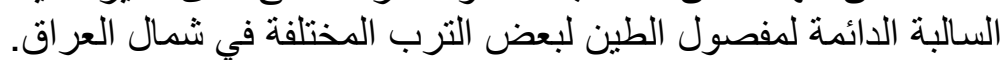

\section{مواد البحث وطر ائقه}

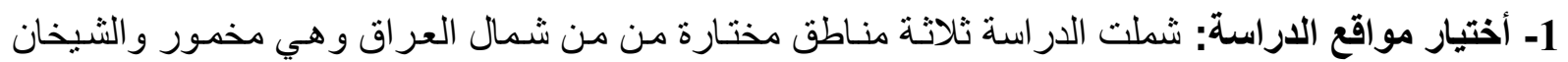

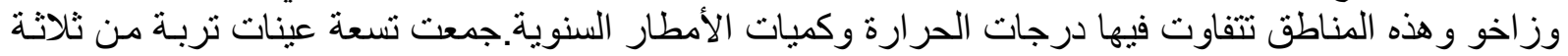

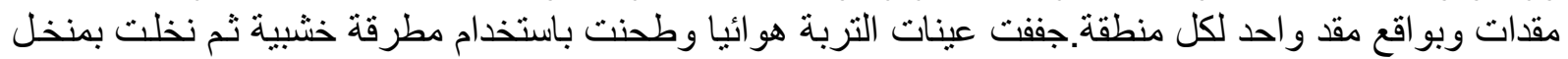
(2ملم) وحفظت العينات في علب بلاسنتيكية محكمة الغلق لكن

2- فصل الطين Separation of Clay: تم فصل الطين عن مكونات التربة الأخرى بدون معاملة التربـة بأيسة

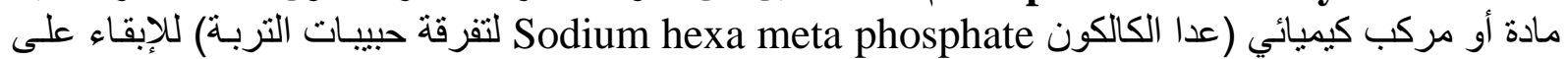

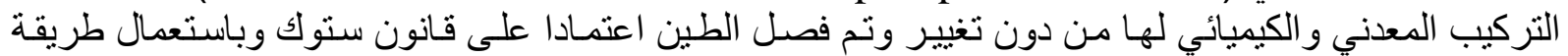

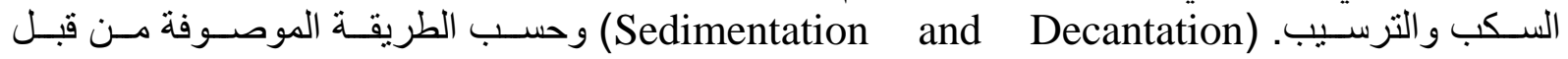
(1979 ، Jackson)

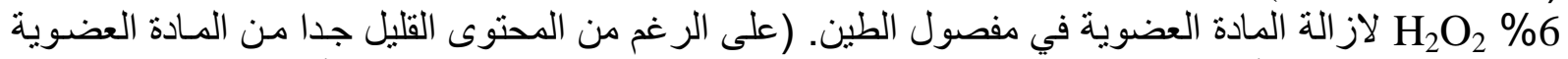

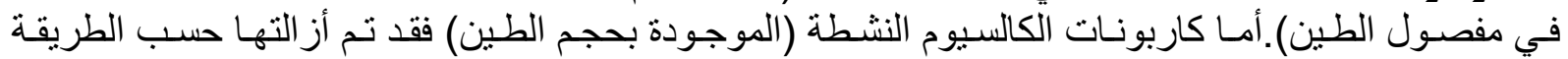

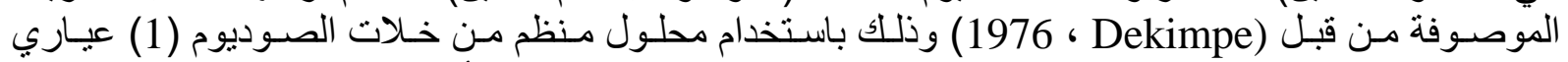

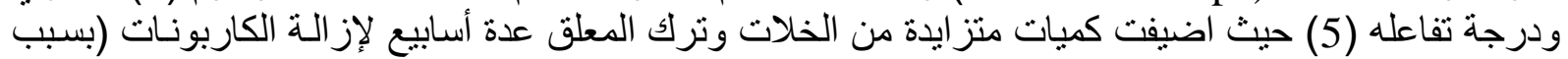

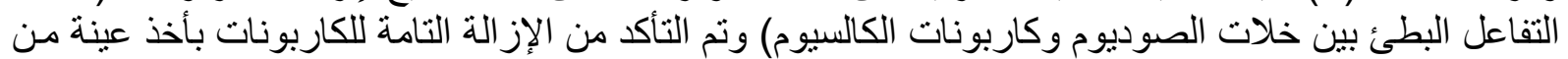

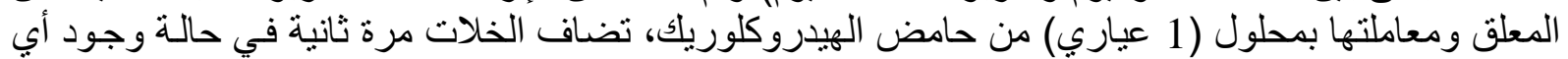

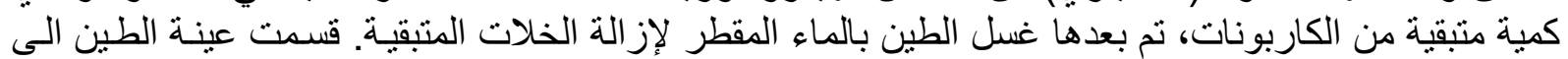

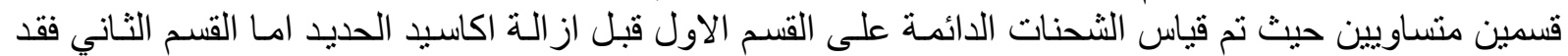

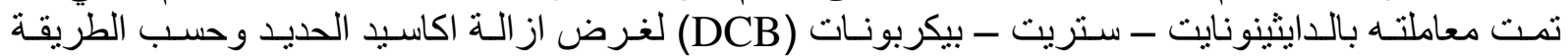

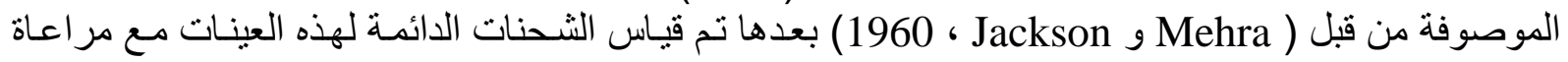
التغير في وزن العينة عند احتساب كمية الثحنات السالبة.

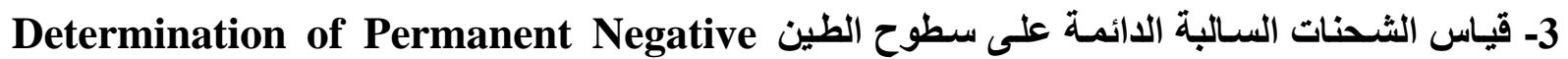
كلورةCharge on Clay Surfaces كلوريد الليثيوم، حيث يمثل الليثيوم الممتز كمية الثحنات السالبة الدائمة على سطوح الطين، الطين، أمـا الكلوريد الممتز 


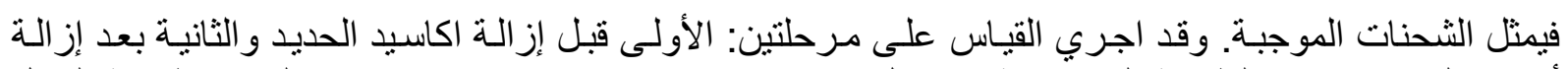

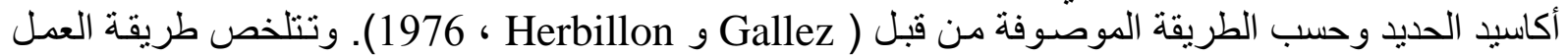

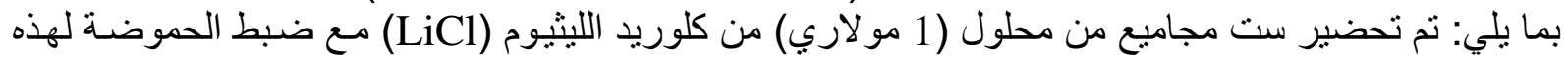

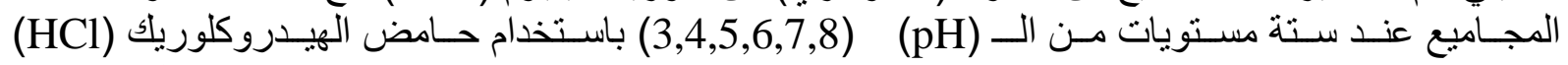

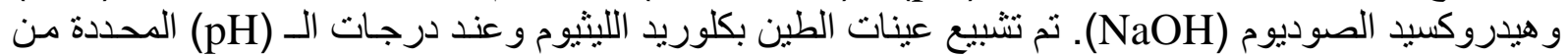

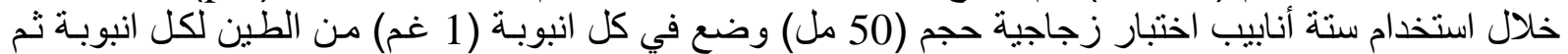

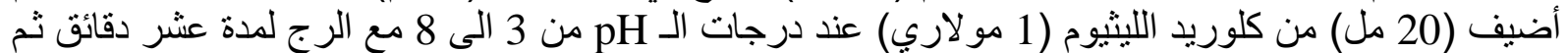

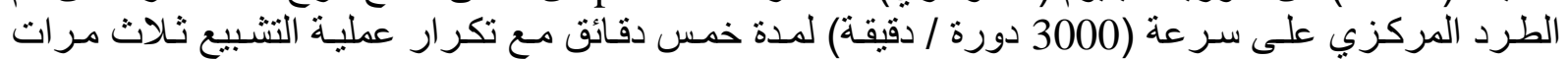

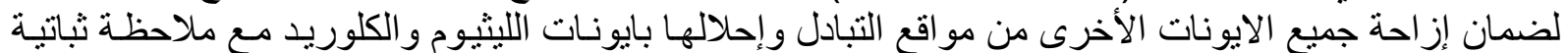

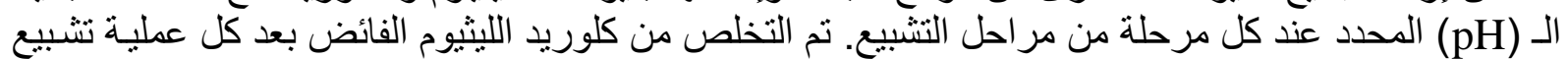

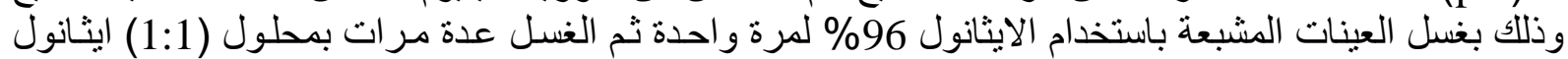

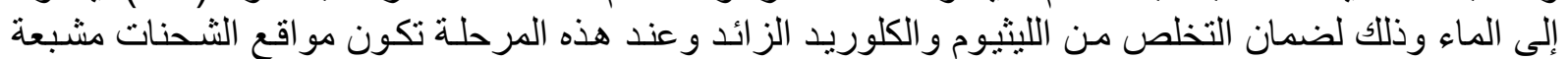

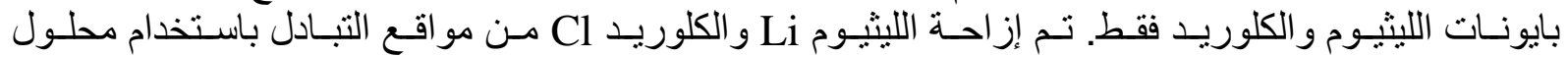
(1 مـو لاري) مـن خـلات الامونيـوم (CH

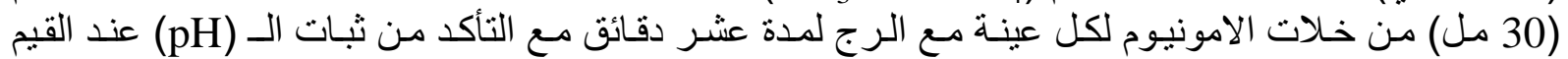

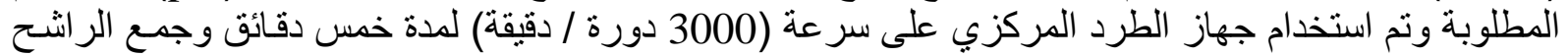

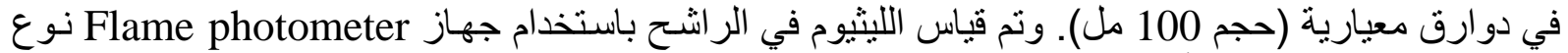
Shewood

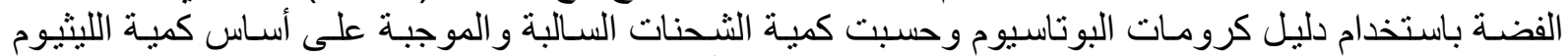

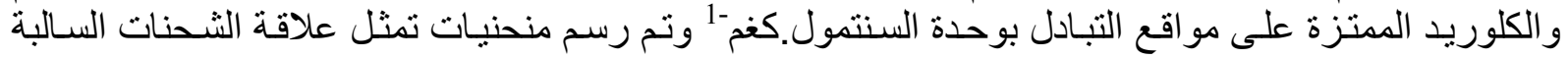
و الموجبة مع قيم الـ (pH) حيث تعبر كمية الليثيوم الممتز عن كمية الثحنات السالبة الدائمـة بينمـا كميـة الكلوريد المتز الثحنات الموجبة.

\section{النتائج والمناقشة}

1- تقدير أكاسيد الحديـ Determination of Iron Oxides: تراوحت نسبة أكاسـبد الحديد الحرة (Free Iron Oxides)

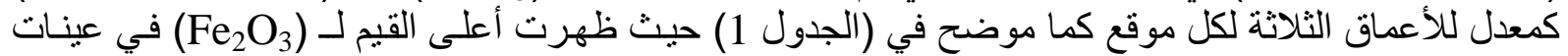

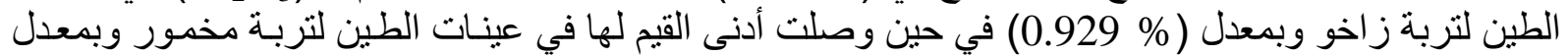

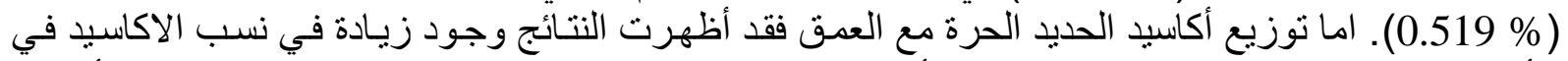

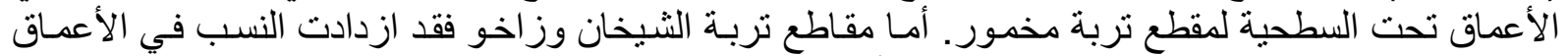

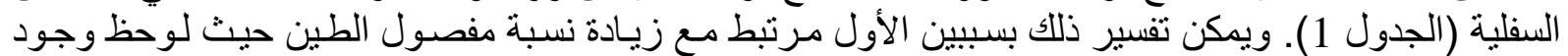

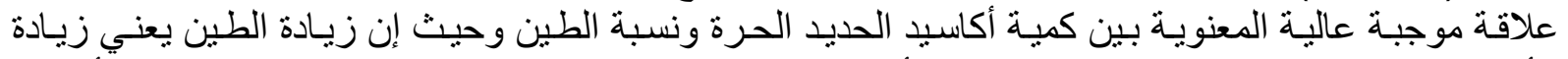

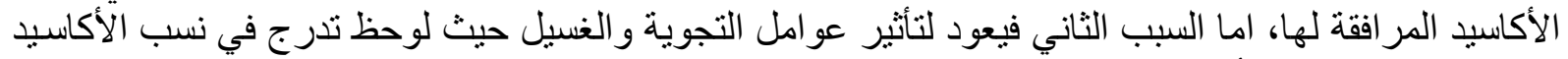

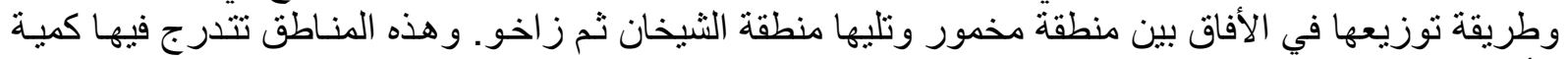

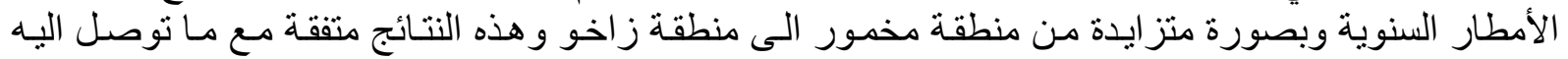

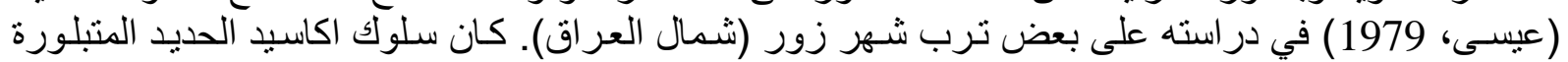
وأكاسيد الحديد غير المتبلورة (Crystalline Fe Oxide)

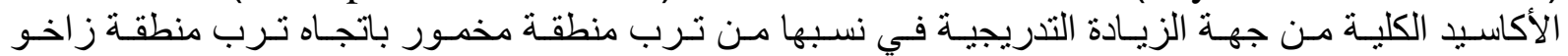

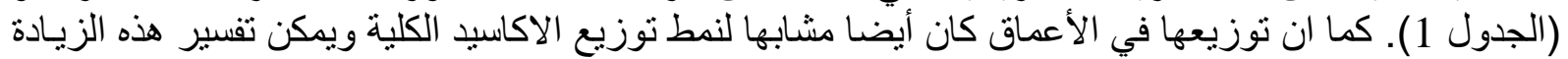

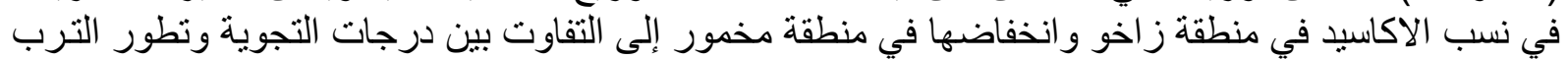

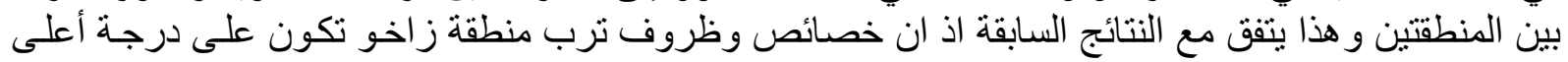

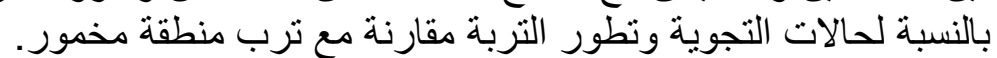

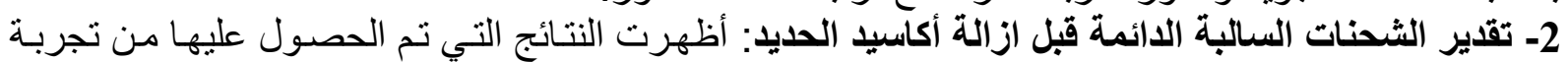

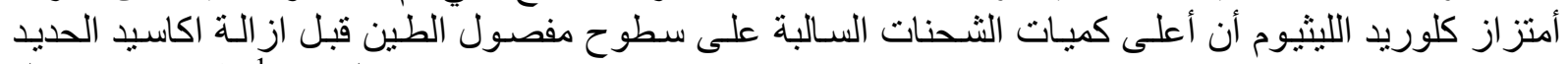

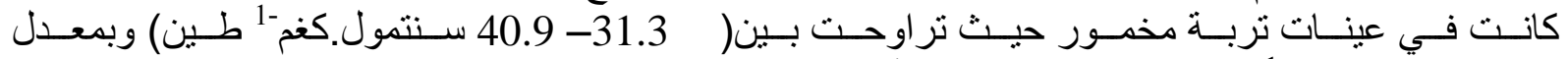

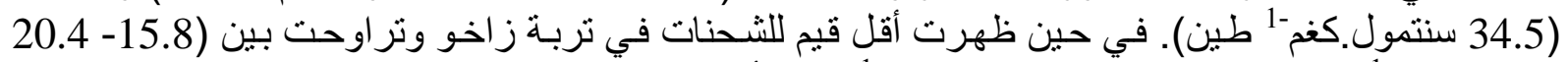

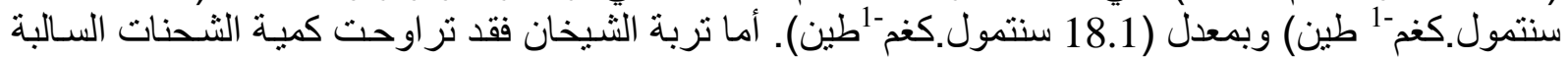


بين (19.2- 21.5 سنتمول.كغف-طين) وبمعدل (20.0 سنتمول.كغم-طين) (الجدول1)، أمسا مديات استقر ارية

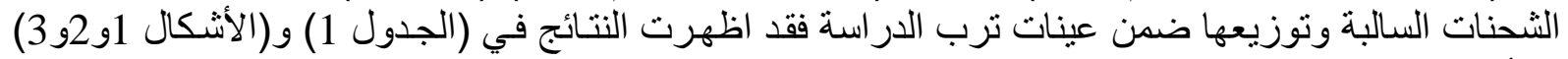

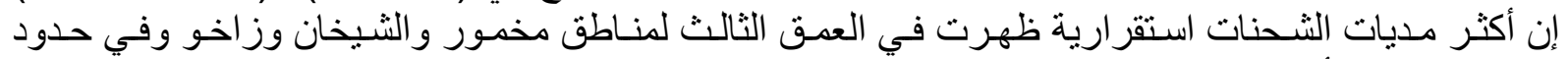

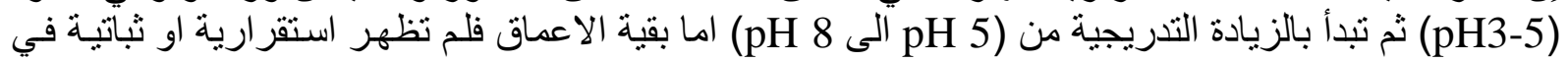

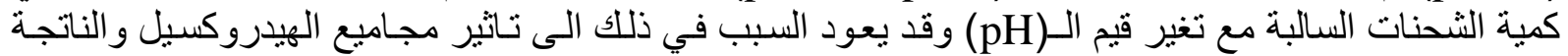

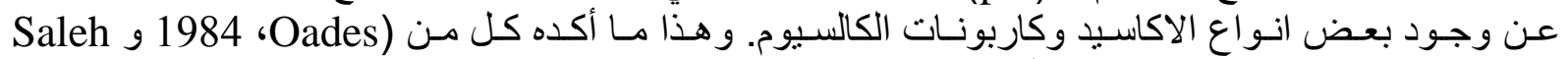

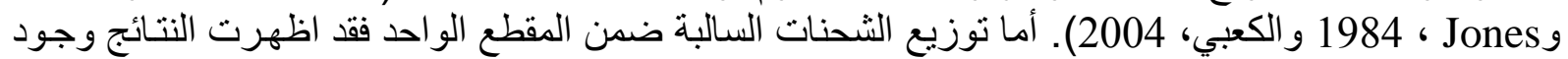

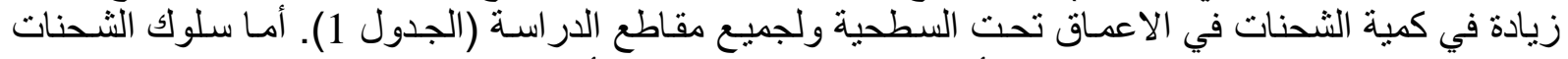

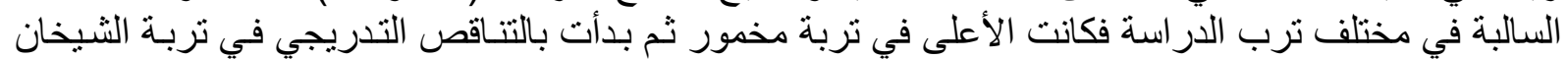

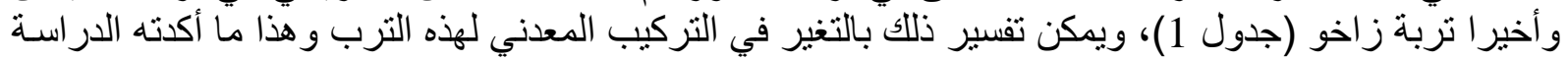

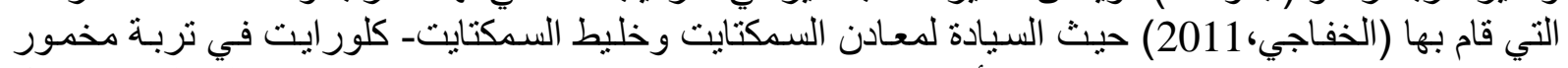

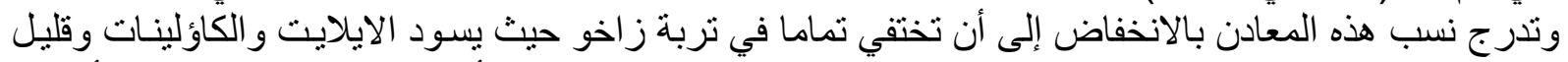

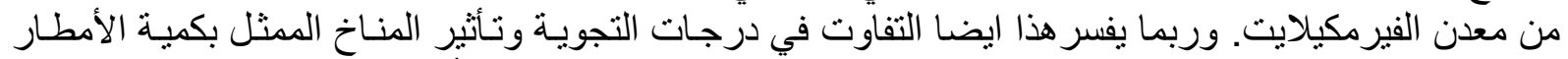

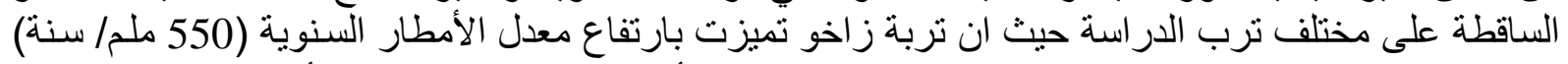

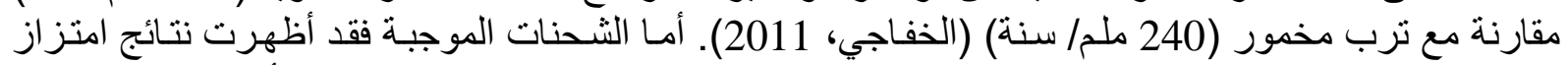

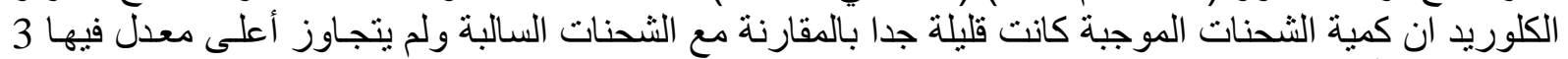

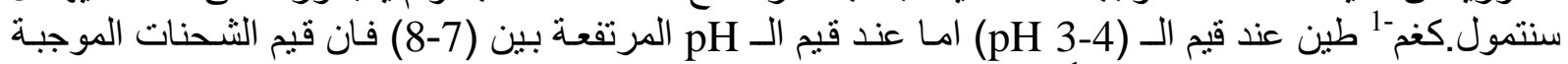

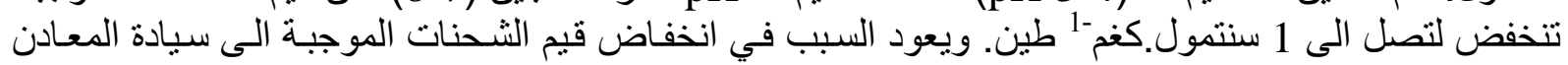

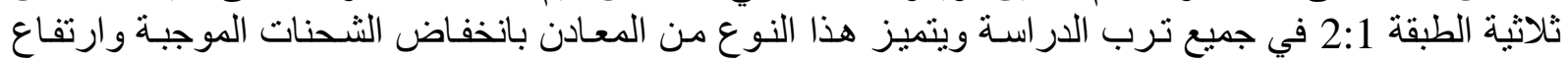

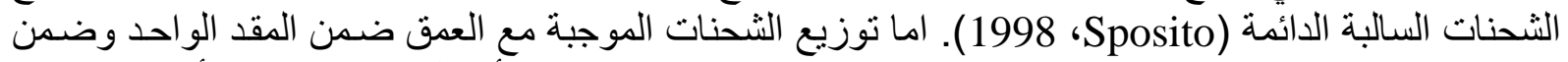

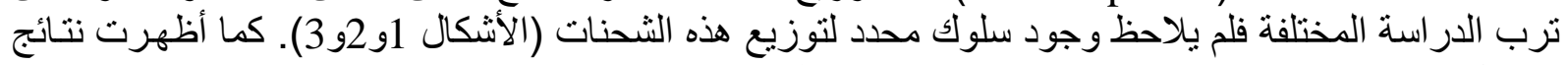

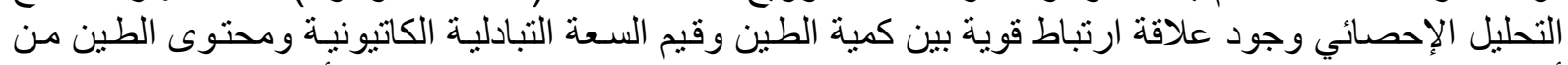

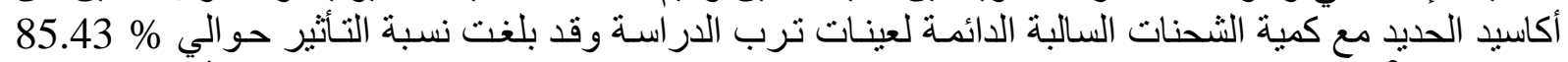

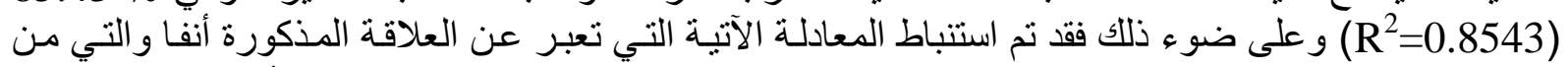

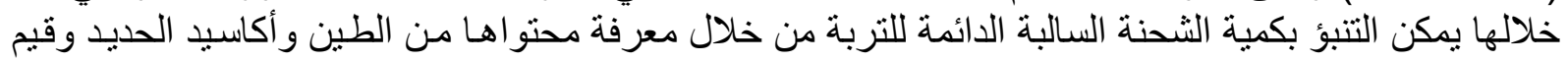
السعة التبادلية الكاتيونية وكما بلية بلية

$\mathrm{NSC}(\mathrm{P})=42.166+0.043 \times($ Clay $)-1.088 \times(\mathrm{CEC})-19.695 \times\left(\mathrm{Fe}_{2} \mathrm{O}_{3}\right)$

NSC (P): Negative Surface Charges (Predict)

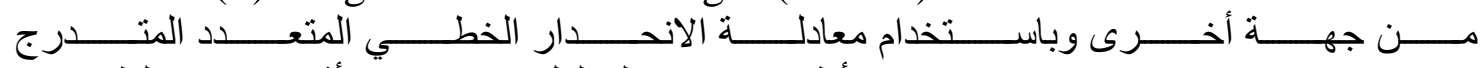

أظهرت نتائج التحليل الإحصـائي تأثثير محتوى الطين من النئ (Step Wise Multiple Linear Regression)

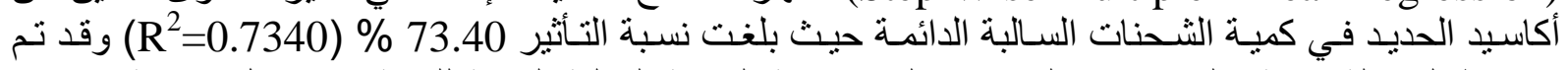

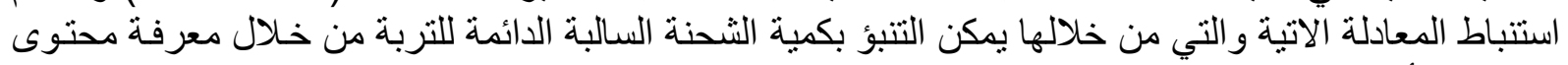

$\mathrm{NSC}(\mathrm{P})=55.45-39.65 \times\left(\mathrm{Fe}_{2} \mathrm{O}_{3}\right)$

3- تقلير الثحنات السالبة الائمة بعد ازالة أكاسيد الحديد: أظهرت نتائج امتز از كلوريد الليثيوم على عينات

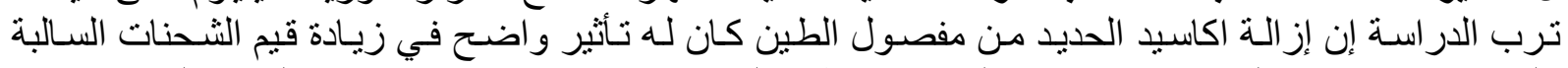

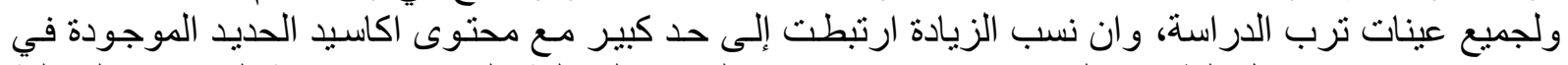

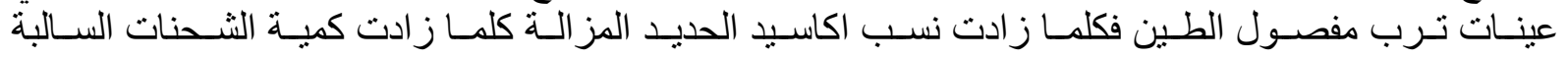

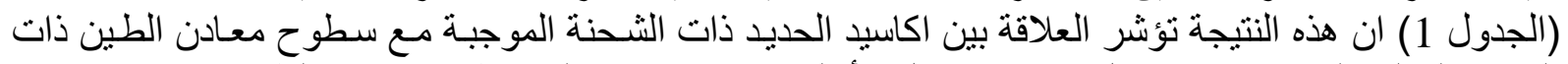

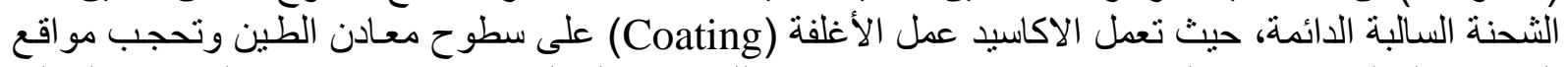

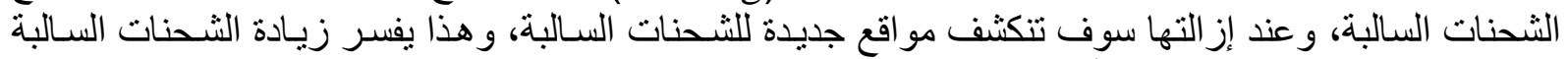

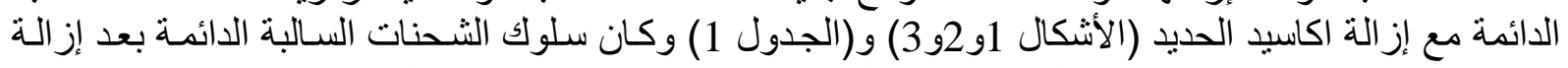

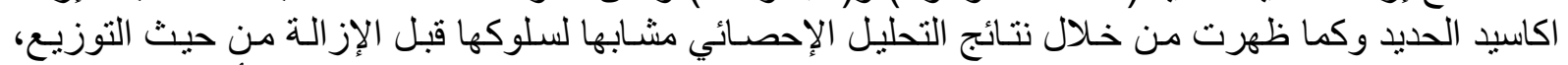

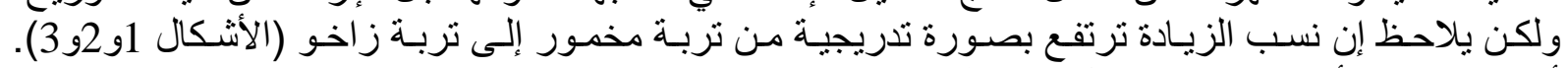

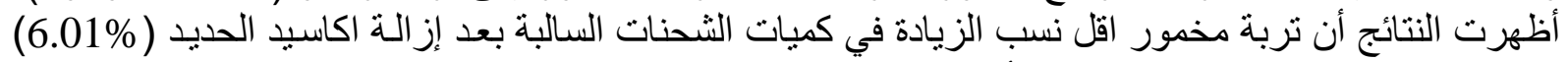

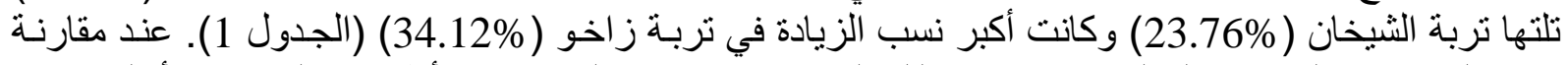

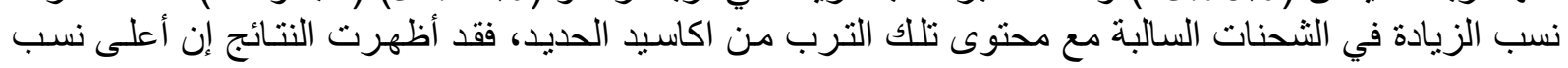


اكاسبد الحديد كانت في تـرب زاخو (0.929\%) (الجدول 1) في حين كانت اقل النسب في نربـة مخمور

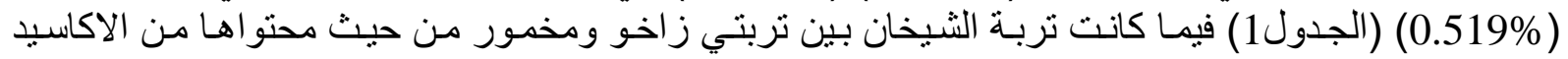

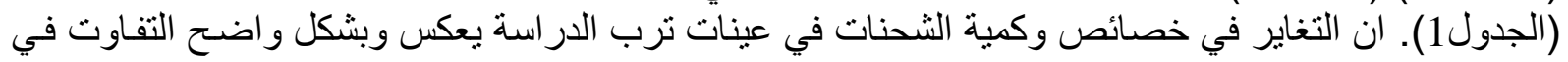

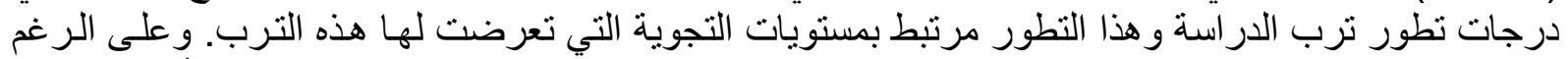

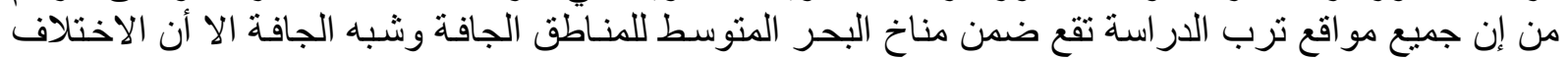

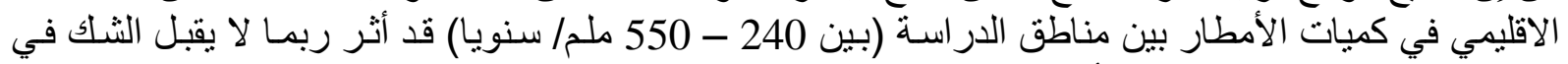

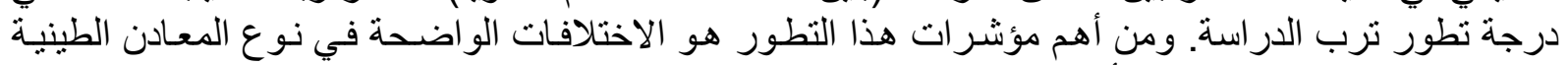

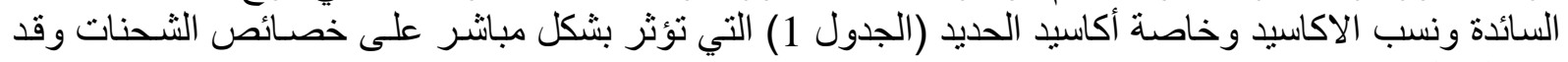

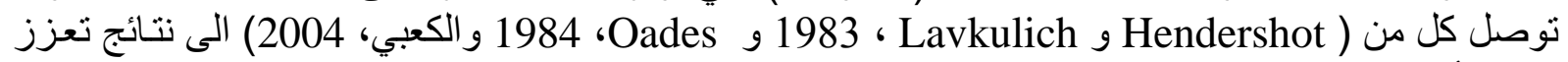
النتائج أعلاه في در اسات مختلفة على خصـائص الثـحنات. وكذللك تبين من نتائج التحليل الإحصـائي باستخدام

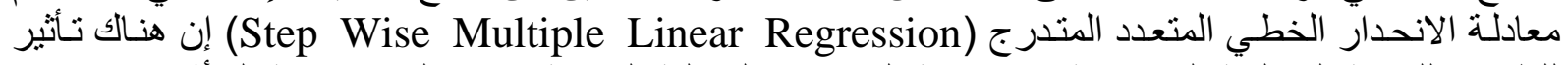

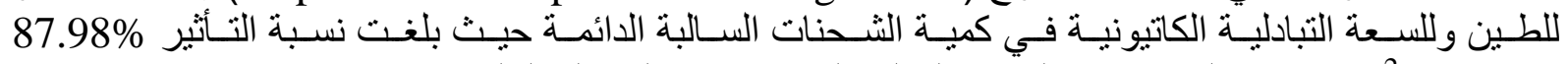
(R²=0.8798) ويمكن التنبؤ بكمية الثنحنة السالبة الدائمة حسب النئ المعادلة التالية: $\mathrm{NSC}(\mathrm{P})=36.67+0.043 \times($ Clay $)-1.319 \times(\mathrm{CEC})$
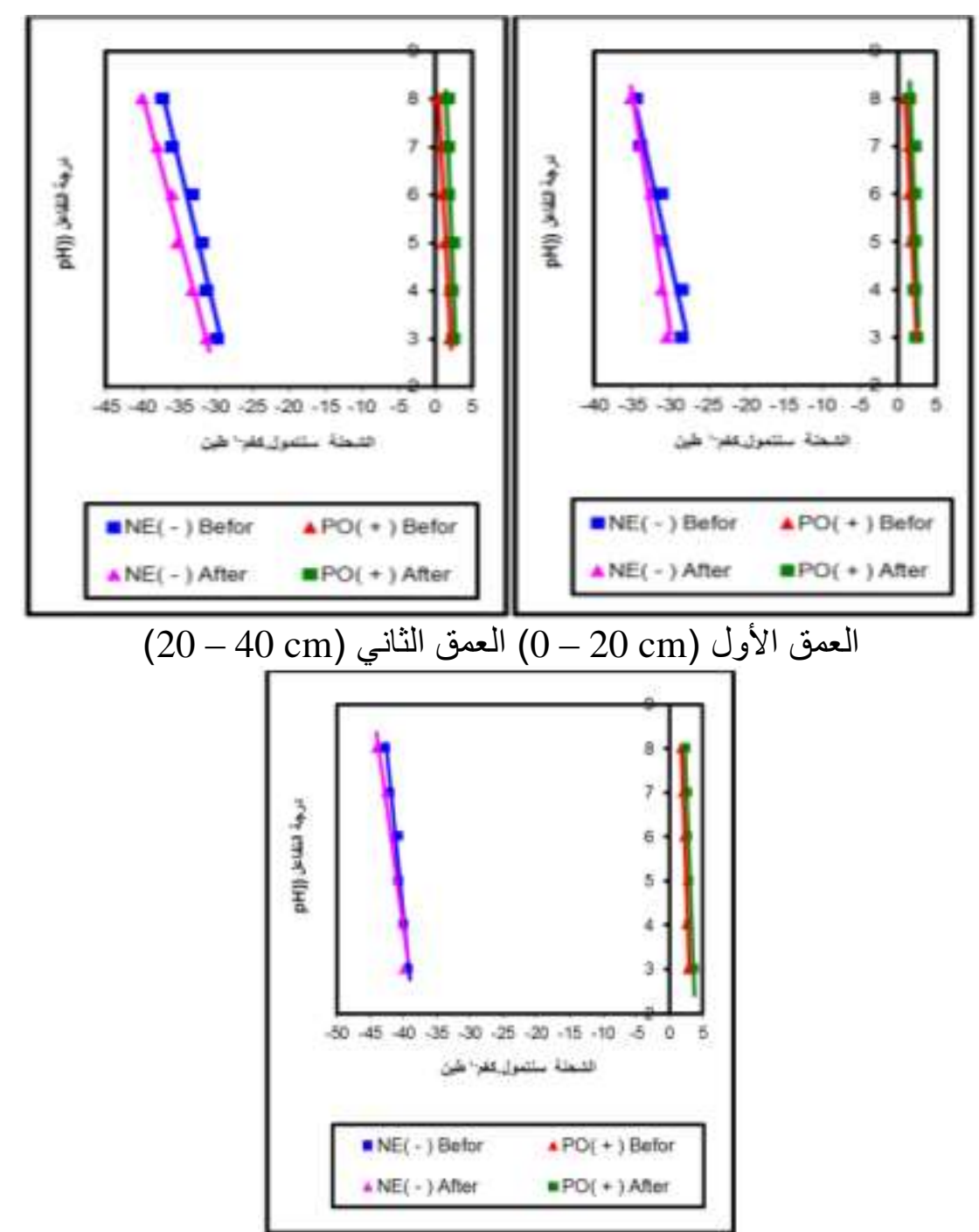

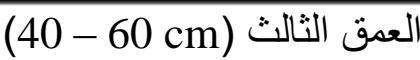

الثـكل (1): كميـة الثـحنات السـالبة والموجبـة لعينـات الطين في منطقة الثـة مخمور و المقدرة مـن امتزاز الليثيوم والكلوريد قبل وبعد ازالة أكاسيد الحديد

Figure (1): Permenant negative and positive charge for clay samples for makhmour area measured by adsorption of LICL before and after removal of iron oxide 


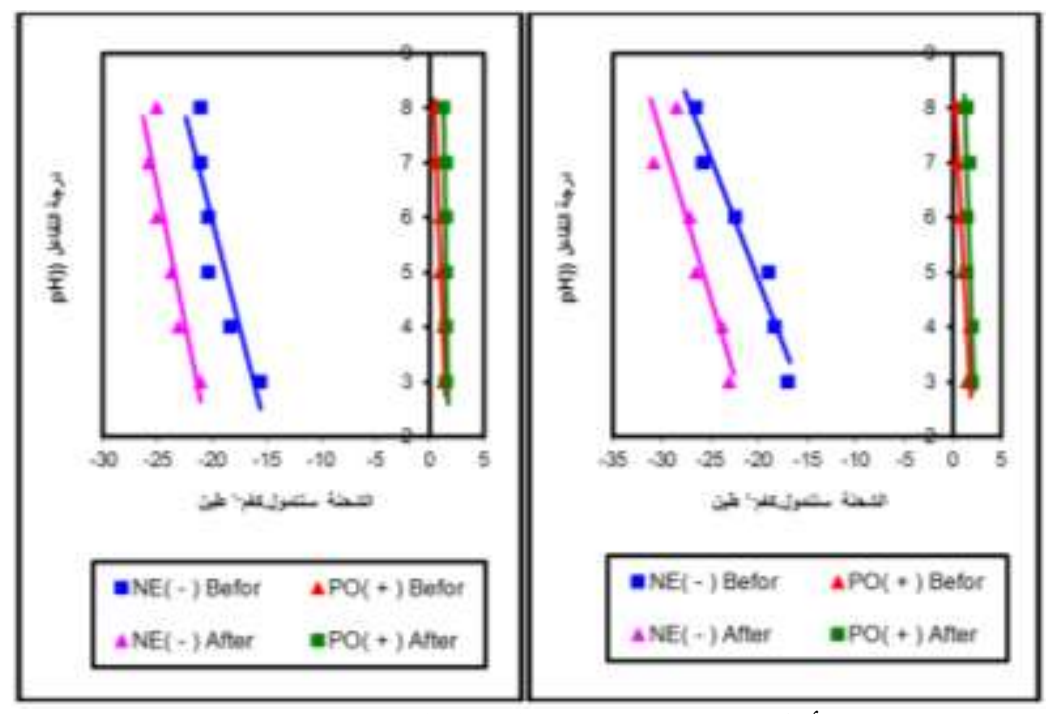

العمق الأول (20 cm 40 cm) العمق الثاني (0)

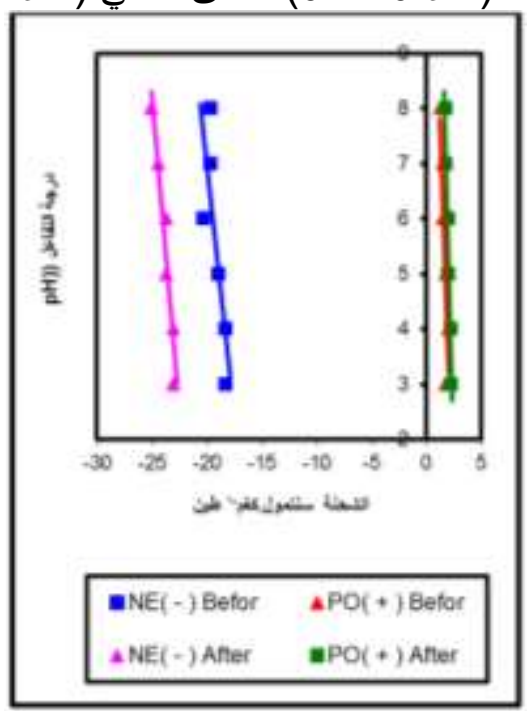

العمق الثالث (40m 60 - 60 (

الثكل (2): كمية الثحنات السالبة و الموجبة لعينات الطين في منطقة الثيخان و المقدرة من امتزاز الليثيوم والكلوريد قبل وبعد از الة أكاسيد الحديد

Figure (2): Permenant negative and positive charge for clay samples for shikhan area measured by adsorption of LICL before and after removal of iron oxides.

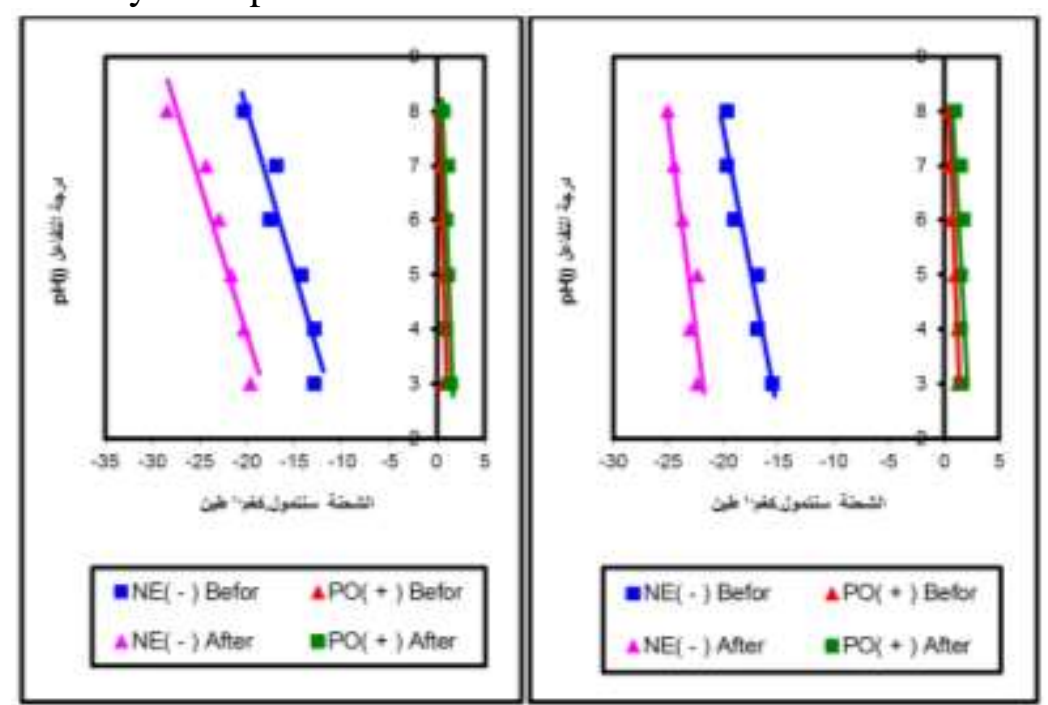

العمق الأول (20 cm 20 - 40 cm) العمق الثاني (20) 
Mesopotamia J. of Agric.

Vol. (45) No. (2) 2017
ISSN: 2224 - 9796 (Online)

ISSN: 1815 - 316 X (Print)

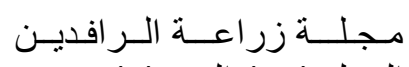

المجلد (45) العدد (2) 2017

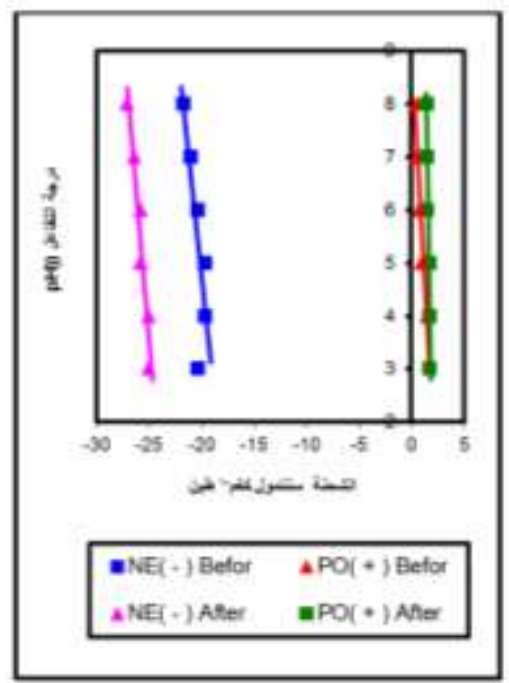

العمق الثالث (40 - 60 cm)

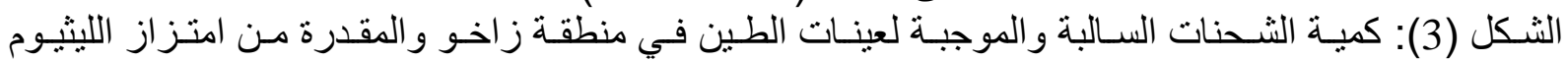
والكلوريد قبل وبعد ازالة الكاسيد الحديد

Figure (3): Permenant negative and positive charge for clay samples for zakho area measured by adsorption of LICL before and after removal of iron oxides.

الجدول (1) : توزيع أكاسيد الحديد الكلية وعلاقتها مع كميات الثحنات السالبة لعينات الطين. Table (1) : Distribution Of Iron Oxides And it's Relation With The Amounts Negative Charge For Clay Samples.

\begin{tabular}{|c|c|c|c|c|c|c|c|c|c|}
\hline \multirow{2}{*}{$\begin{array}{c}\text { المعد } \\
\% \\
\text { Average } \\
\%\end{array}$} & \multirow{2}{*}{$\begin{array}{c}\text { نسبة الزيادة } \\
\text { \% } \\
\text { Increasing } \\
\%\end{array}$} & \multicolumn{4}{|c|}{ 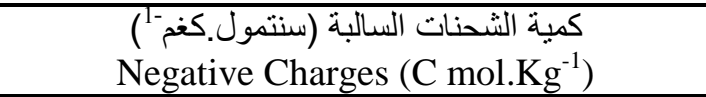 } & \multicolumn{2}{|c|}{ 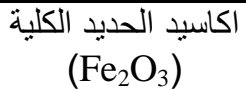 } & \multirow{2}{*}{$\begin{array}{c}\begin{array}{c}\text { العمth } \\
\text { Depth }\end{array} \\
\text { cm }\end{array}$} & \multirow{2}{*}{$\begin{array}{c}\text { عينات } \\
\text { Soil } \\
\text { Samples }\end{array}$} \\
\hline & & $\begin{array}{c}\text { المعدل } \\
\text { Average }\end{array}$ & $\begin{array}{c}\text { بعد الإز الة } \\
\text { After } \\
\text { Removing }\end{array}$ & $\begin{array}{c}\text { المعدل } \\
\text { Average }\end{array}$ & $\begin{array}{c}\text { قبل الإز الة } \\
\text { Before } \\
\text { Removing }\end{array}$ & $\begin{array}{c}\text { المع } \\
\text { (\%) } \\
\text { Average }\end{array}$ & $\%$ & & \\
\hline \multirow{3}{*}{6.01} & 2.52 & \multirow{3}{*}{36.35} & 32.05 & \multirow{3}{*}{34.43} & 31.26 & \multirow{3}{*}{0.519} & 0.502 & $0-20$ & \multirow{3}{*}{ 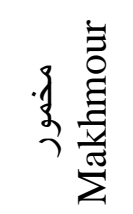 } \\
\hline & 14.14 & & 35.59 & & 31.18 & & 0.548 & $20-40$ & \\
\hline & 1.39 & & 41.42 & & 40.85 & & 0.508 & $40-60$ & \\
\hline \multirow{3}{*}{23.76} & 23.29 & \multirow{3}{*}{24.76} & 23.92 & \multirow{3}{*}{20.0} & 19.40 & \multirow{3}{*}{0.852} & 0.822 & $0-20$ & \multirow{3}{*}{ 雪 } \\
\hline & 23.88 & & 26.56 & & 21.44 & & 0.862 & $20-40$ & \\
\hline & 24.13 & & 23.81 & & 19.18 & & 0.874 & $40-60$ & \\
\hline \multirow{3}{*}{34.12} & 45.02 & \multirow{3}{*}{24.07} & 22.90 & \multirow{3}{*}{18.05} & 15.79 & \multirow{3}{*}{0.929} & 0.908 & $0-20$ & \multirow{3}{*}{$\frac{7}{1} \frac{\frac{2}{n}}{\frac{N}{N}}$} \\
\hline & 30.82 & & 23.47 & & 17.94 & & 0.925 & $20-40$ & \\
\hline & 26.54 & & 25.84 & & 20.42 & & 0.954 & $40-60$ & \\
\hline
\end{tabular}




\title{
SIGNIFICANCE OF SOIL DEVELOMENT AND IRON OXIDES CONTENT ON PROPERTIIES OF PERMENANT CHARGE ON CLAY SURFACES IN SOME SELECTED SOILS FROM NORTHERN IRAQ
}

\author{
Saleh A. Mawlood \\ Qahtan D. Essa \\ Soil Science \& Water Resources Dept., College of Agriculture and Forestry, \\ Mosul University. Iraq \\ E-mail: Adel_mawlood@yahoo.com
}

\begin{abstract}
The study was conducted on nine clay samples collected from three different soils of northern Iraq (Mukhmour, Shikhan and Zakho) which varied in climatic conditions (precipitations and temperatures).Permanent surface charge on clay surfaces was measured through adsorption of lithium chloride experience before and after removing iron oxides. Results showed that the highest values of permanent negative charge on clay surfaces before removing iron oxides was found in Mukhmour soil with average 34.5 C.mol. $\mathrm{kg}^{-1}$. clay, while the lowest values appeared in clay fraction of Zakho soil (18.1 C.mol. $\mathrm{kg}^{-1}$. clay), but in Shikhan soil,the negative charge values was at average of (20.0 C.mol. $\mathrm{kg}^{-1}$ clay). The highest stability of negative surface charge was found at $\mathrm{pH}$ between values (3-5). The distribution of negative charges within soil profiles showed an increase in values with depth and in all studied soils. Results also reveals very low positive surface charge compared with negative surface charge. However, the results of the lithium chloride adsorption experiment have indicated that the removal of iron oxides from clay fraction had a clear effect on increasing in the values of negative surface charge in all study soils. The rates of increase associated mainly with the iron oxides content of the clay fractions but the impact of removing iron oxides on the values of positive surface charge were minor and had no clear behavior. From the above study it can be concluded that the variations in the values of surface of clay surface charge clearly reflects the levels of weathering and the degrees of soil development.
\end{abstract}

Keywords: positive surface charge, negative surface charge, Iron Oxides.

Received: 6/12/2012, Accepted: 18/2/2013.

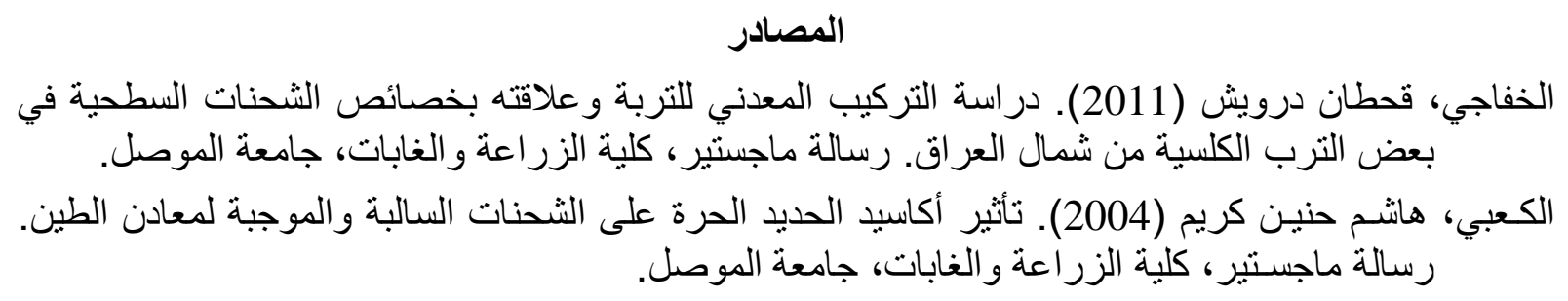

Bouman, O.T., D. Curtin., C.A. Campbell., V.O. Biederbeck, and H. Ukrainetz, (1995). Soil acidification from long-term use of anhydrous ammonia and Urea. Soil Science Society of American Journal. 59:1488-1494.

Curtin, D., Rostad, H.P.W. and P.M. Huang (1984). Soil acidity in relation to soil properties and lime requirement. Candian Journal of Soil Science. 64:545-554.

Dekimpe, R. C. (1976). Mineralogical analysis. In J. A. Mckeague (ed.) Manual On Soil Sampling Method Of Analysis. Soil Research Institute, Ottawa. P.203-287. 
Gallez, A.S., Juo, R. and A.J. Herbillon (1976). Surface and charge characteristics of selected soils in the tropics. Soil Science Society of American Journal. 40:601608.

Ge, Y. and W. Hendershot (2004). Evaluation of soil surface charge using the backtitration technique. Soil Science Society of American Journal: 68:82-88.

Hendrshot, W. H. and L. M. Lavkulich (1983). Effect of sesquioxides coatings on surface charge of standard mineral and soil samples. Soil Science Society of American Journal. 47: 1252-1260

Jackson, M. L. (1979). Advance soil chemical analysis cource-ed.2.

Karlen, D.L. and D.E. Stott (1994). A frame work evaluating physical and chemical Indicators of soil quality. P. 53-72 in J.Doran, D.C.Coleman.D.F.

Mehra, O. P. and M. L. Jackson (1960) Iron oxide removal from soils and clay by a dithionite citrate system buffered with sodium bicarbonate. Clays Clay Minerals: 7, $317-327$.

Oades, J. M. (1984). Interaction of polycations of aluminum and iron with clay. Clay Miner. 32: 49-57.

Rhoades, J.D. (1982). Cation exchange capacity P. 149-157 in A.L. Page (ed) Methods of Soil Analysis part 2, $2^{\text {nd }}$ ed. Madison, Wisconsin.

Saleh, A. M. and A. A. Jones (1984). The crystallinity and surface characteristics of synthetic ferrihydrite and its relationship to kaolinite surface. Clay Minerals 19:745-755.

Sanchez, P. A. and T. J. Logan. (1992). Myths and science about the chemistry and Fertility of soils in the tropics. P. 35-46. In Lal and P.A. Sanchez. (ed) SSSA Spec. Publ. 29, Madison, Wisconsin.

Sparks, D.L. (1995). Environmental Soil Chemistry. Academic Press, Inc. New York.

Sposito, G. (1998). On points of zero charge. Environmental Science Technology 32:2815-2819.

Uehara, G. and G. Gillman (1981). The Mineralogy, Chemistry, and Physics of Tropical Soils with Variable Charge Clays. 
Mesopotamia J. of Agric.

Vol. (45) No. (2) 2017
ISSN: 2224 - 9796 (Online)

ISSN: 1815 - 316 X (Print)

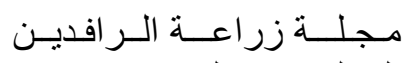

المجلد (45) العدد (2) 2017 